\title{
I. Eröffnung der Tagung am 15. Oktober I953
}

\section{Eröffnungsansprache des zweiten Vorsitzenden Professor Dr. Ipsen}

Herr Prorektor, Spektabilität, verehrte Gäste, liebe Kollegen!

Ich habe die Ehre, Sie zur I2. Tagung der Vereinigung deutscher Staatsrechtslehrer hier in Bonn willkommen zu heißen. Wie vor einem Jahre in Marburg muB ich zu meinem Bedauern mitteilen, $\mathrm{da} B$ unser verehrter Kollege Wolff, dem dieser Platz hier gebührt, wiederum nicht imstande ist, an unserer Tagung teilzunehmen, weil ihn seine Erkrankung noch ans Bett fesselt und wohl erst in der nächsten Woche die Hoffnung besteht, da $B$ er aus seiner Lage, die ihn seit einem Jahr behindert, befreit wird. Ich darf Ihr Einverständnis damit annehmen, daB ich Herrn Wolff in einem Grußtelegramm Ihre Besserungswünsche übermittle, in der Hoffnung, $\mathrm{daB}$ wir ihn sehr bald wieder in unserem Kreise begrüßen dürfen. Herr Wolff seinerseits hat, abgesehen von den Vorbereitungsarbeiten für die Tagung, die er in keiner Stunde vernachlässigt hat, unser gedacht in einem Telegramm, das ich hier zur Verlesung bringen darf:,, Auswärts und ganz ohne Gewalt gedenke ich derer, die auf die Sozialität unseres Rechtsstaats bedacht. Hans Wolff"'.

Ich habe die Ehre, in unserem Kollegen Friesenhahn den Prorektor der Rheinischen Friedrich-Wilhelms-Universität Bonn zu begrüßen, und darf ihn bitten, zunächstdas Wort an uns zu richten.

Begrüßungsworte des Prorektors der Universität Bonn Professor Dr. Friesenhahn

Meine verehrten Herren!

In Vertretung seiner Magnifizenz, Professor Dr. Richter, der heute morgen zur Tagung der Forschungsgemeinschaft und der Rektorenkonferenz nach Bremen abgefahren ist, habe ich die große Ehre, Sie hier als Hausherr willkommen zu heißen und Ihnen die herzlichsten Grüße und Wünsche von Rektor und Senat der Rheinischen Friedrich-Wilhelms-Universität zu entbieten. Drei Jahre nach der Begründung der Weimarer Republik trafen sich die deutschen Staatsrechtslehrer in der Friedrich-Wilhelms-Universität der Reichshauptstadt Berlin, um ihre Vereinigung zu gründen. Die Schlesische Friedrich-Wilhelms-Universität in Breslau sollte der Ort der Tagung sein, die dann, nach Halle verlegt, die letzte im ersten Lebensabschnitt unserer Vereinigung - ich 
muB jetzt sagen: Ihrer Vereinigung - gewesen ist. Sie kommen jetzt in die letzte noch bestehende Friedrich Wilhelms-Universität und kommen damit zugleich in eine Stadt, die zur vorläufigen Bundeshauptstadt erhoben ist, und die dem Grundgesetz den Namen gegeben hat, um dessen Auslegung Sie sich mühen. Rektor und Senat der Universität freuen sich, Ihrer Tagung einen würdigen Rahmen in unserer wieder aufgebauten Universität bieten zu können. Ich darf Ihnen sagen, daB dieser Raum, in dem die alten Bonner wohl kaum unsere alte Aula wiedererkennen, durch diese Tagung seine Weihe erfährt. Er ist in den allerletzten Tagen erst fertig geworden. Und so darf ich nun Ihrer Tagung den besten Erfolg wünschen. Sie haben sich grundlegende Fragen unserer Staatsgestaltung als Thema Ihrer Beratungen gewählt. Ich darf wünschen, $\mathrm{da} B$ gerade aus diesen Beratungen in der Universität der Bundeshauptstadt nicht nur für Sie selbst neue Erkenntnisse wachsen, sondern daß Ihre Beratungen auch segensreich sein mögen für Volk und Staat.

\section{BegrüBungsworte des Dekans der Rechts- und Staats- wissenschaftlichen Fakultät der Universität Bonn Professor Dr. Scheuner}

Meine sehr verehrten Kollegen!

Ich darf Sie namens der Rechts- und Staatswissenschaftlichen Fakultät der Rheinischen Friedrich-Wilhelms-Universität hier herzlich willkommen heißen. Wir freuen uns, daß in diesem Jahre die Wahl der Staatsrechtslehrervereinigung auf Bonn gefallen ist, und wir sind um so glücklicher, da $B$ wir Sie hier in einem neu hergerichteten Gebäude empfangen können, das zwar noch vielfach die Spuren des Krieges trägt, das aber doch nun gewissermaßen wie der Phönix aus der Asche sich zu neuem Glanze erhoben hat. Besonders aber freue ich mich, dab wir an diesem Tage auch noch einige Kollegen der Vereinigung der Zivilrechtslehrer hier begrüßen können, die ich ebenfalls herzlichst willkommen heiße.

\section{Professor Dr. Ipsen erwidert:}

Herr Prorektor, Spektabilität!

Ich darf Ihnen aufrichtig danken für Ihre BegrüBungsworte und damit verbinden ein besonders herzliches Wort des Dankes einmal dafür, daß uns diese Räume nach ihrer Neugestaltung für unsere Tagung zur Verfügung gestellt worden sind, darüber hinaus und nun bin ich mir im Zweifel, inwieweit ich den Herrn Prorektor und seine Spektabilität oder die Kollegen Friesenhahn und Scheuner ansprechen darf - danken für die mühseligen Vorbereitungsarbeiten, die beide hier am Ort geleistet haben, um der 
Tagung den Rahmen und die Ermöglichung zu geben, die ihr schlieBlich zuteil geworden ist. Ich darf meinen Dank erstreckenund Herrn Friesenhahn freundlichst bitten, ihn zu tibermitteln auf Frau Friesenhahn, die die Liebenswïrdigkeit hatte, sich unserer Damen anzunehmen, nicht zuletzt auch meinen Dank richten an unseren Kollegen Köttgen, der einmal in uneigennützigster Weise es übernommen hatte, bei der Programmgestaltung erster Úberlegung in einem Vortrag, den wir als Gastgeber veranstalten wollten, zu sprechen, und dann nicht minder uneigennützig sich den Notwendigkeiten des Tages und der schnellen Entscheidung anpaßte, indem er ohne weiteres zurïcktrat von diesem Vorhaben. Ich hoffe, $\mathrm{da} B$ er die mühselige Vorbeteitung seines Vortrages nunmehr in einem späteren Zeitpunkt auch hier in Bonn zur Geltung gebracht sehen wird.

Meine verehrten Herren! Die Gunst des Ortes vermittelt uns die besondere Freude und Ehre, da $B$ an dieser Tagung auch teilzunehmen imstande ist der lebenslängliche Ehrenpräsident unserer Vereinigung, unser verehrter Kollege Herr Geheimrat Thoma, den ich nun bitten darf, $z \mathfrak{u}$ uns $z u$ sprechen.

\section{Ansprache des Ehrenpräsidenten}

Geheimen Hofrats Professor Dr. Richard Thoma

\section{Meine sehr verehrten und lieben Herren Kollegen!}

Ihr dankenswerter EntschluB, die diesjährige Tagung in der Bundeshauptstadt abzuhalten und damit an dem Sitze der Universität, der ich seit nunmehr 25 Jahren angehöre, gibt mir die erwünschte Möglichkeit, auch einmal persönlich vor Sie zu treten in der Wtirde, mit der Sie mich so gütig ausgezeichnet haben, als Ehrenpräsident der Vereinigung der deutschen Staatsrechtslehrer.

Gestatten Sie mir, die Tagung mit einem Geleitwort einzuleiten. Als wir uns nach den Jahren der Gewaltherrschaft und des Krieges zum ersten Male wieder versammelten, in Heidelberg im Oktober 1949, da waren wir uns einig in dem Wunsche und in der Zuversicht, daß es der Vereinigung gelingen werde, eine Arbeit zu leisten, die sich den Leistungen der ersten Io Jahre ihrer Tätigkeit würdig zur Seite stellt. Ist diese Erwartung erfüllt worden ? Es wäre, meine verehrten Herren Kollegen, unwahrhaftig, zu verschweigen, da $B$ gegen die eine oder andere Einzelleistung gewichtige Worte der Kritik laut geworden sind. Aber es kommt auf das Ganze an. Der Gesamtüberblick über die 4 Tagungen, die seitdem stattgefunden haben, berechtigt, wie mir scheint, dazu, eine Bilanz zu ziehen, die ebenso erfreulich wie für die Zukunft verheiBungsvoll ist. Und dazu möchte ich Sie beglückwünschen. Und beglückwïnschen möchte ich Sie auch zu der Wahl der Probleme, mit denen die jetzt beginnende Tagung und ihre Verhandlungen sich beschäfti- 
gen sollen. Das Thema „Begriff und Wesen des sozialen Rechtsstaates" führt in den Mittelpunkt der politisch wirkungsvollsten Bemühungen dentscher Staatsrechtswissenschaft. Meine Gedanken schweifen zurlick in die Zeit, da ich - mehrere Jahre vor dem ersten Weltkrieg - eine Analyse des Rechtsstaats zum Gegenstand einer akademischen Antrittsvorlesung gemacht und mit Entschiedenheit gefordert habe, da $B$ sich die deutsche Verwaltungsrechtswissenschaft in den Dienst der Ideale des Rechtsstaats stellen solle. Schon damals war die Aufgabe sichtbar geworden, neben den Freiheitsverbürgungen und den Sicherungen der GesetzmäBigkeit der Verwaltung auch die fürsorgende Betreuung der Bevölkerung, in die festen Ordnungen des Rechtsstaates einzugliedern. Heute ist dies ein vornehmstes Anliegen der Politiker und der Juristen. Jene Gesinnung und Betätigung, die man zur Zeit der großen Französischen Revolution als Fraternité bezeichnete, allerdings aber neben der Liberté und Egalité vernachlässigte und im Schatten stehen ließ, jene Fraternité bezeichnen wir heute als soziale Hilfsbereitschaft und Reformwilligkeit und in resignierender Einsicht in die leere Relativität jeder Forderung von Gerechtigkeit schlechthin reden wir determinierend von der sozialen Gerechtigkeit, die damit allerdings als eine von mehreren bezeichnet wird. Der Drang zur Verwirklichung sozialer Gerechtigkeit ist die, man darf wohl sagen segensreichste Auswirkung des allgemeinen und gleichen Wahlrechts, aber sie stößt auf andere Gerechtigkeiten, und es entstehen Konflikte, und eben deshalb ist es ebenso notwendig als interessant und eine besonders glückliche Wahl des Themas, zu untersuchen, wie über der Spannung zwischen dem liberalen Rechtsstaat und der sozialen Demokratie der Bogen der Synthese gewölbt werden kann.

Von hoher Bedeutung und belebender Aktualität ist auch das zweite Thema unserer Tagung, das sich beschäftigt mit der verfassungspolitischen Formung der auswärtigen Gewalt. Die hohe außenpolitische Aufgabe, die hier aufklingt, lenkt zugleich den Blick auf eine Bedeutung unserer Arbeit, die die deutsche staatsrechtliche Wissenschaft unserer Epoche emporhebt über diejenige vergangener Jahrzehnte. Denn die außenpolitische Aufgabe unserer politischen Gewaltenträger ist Befolgung einer deutschen Nationalpolitik, die das Heil sucht nicht in nationalistischer Isolierung, sondern in europäischer Gemeinsamkeit. Und dahin führt - die Realitäten lassen keinen anderen Weg zu - notwendig der Weg über eine Wiedererweckung des Deutschen Reiches in Einheit, Freiheit und Friede. Infolgedessen trägt unser gegenwärtiger Verfassungsbau den Charakter des Provisorischen und infolgedessen ist alle Arbeit, die wir der Exegese, der Kritik, der Reform unseres geltenden Bundes- und Landesstaatsrechts widmen, immer zu- 
gleich auch Vorarbeit für die künftige deutsche Nationalverfassung. Noch kann niemand vorhersagen, wann es dem deutschen Volke möglich sein wird, tuber dem ihm von Völkerrechtswegen zukommenden Landgebiet sein Reich zu erneuern und zu ordnen, aber $\mathrm{daB}$ dieser Tag in absehbarer Zeit über dem geängstigten Europa trostreich aufleuchten wird, das glaubt unser Herz und das erstrebt unser Wille. Und von daher gewinnt die Arbeit der deutschen Staatsrechtswissenschaft eine erhöhte Bedeutung und eine besondere Würde. $\mathrm{Zu}$ dieser Arbeit will mein Geleitwort Ihnen Kraft und Gelingen wünschen: bei der Deutung des geltenden Rechts wie bei der Hindeutung auf das künftig zu gestaltende.

Nunmehr bitte ich Herm Professor Ipsen, den Vorsitz zu übernehmen.

\author{
Professor Dr. Ipsen \\ erwidert und beendet die Eröffnungsansprache:
}

Sehr verehrter Herr Ehrenpräsident!

Ich darf Ihnen aufrichtig danken dafür, daß Sie diese einführenden Worte an uns gerichtet haben. Sie werden uns in unserer Arbeit bestimmen, und wir danken dem Augenblick, der uns Ihre Anwesenheit hier geschenkt hat.

In der BegrüBung unserer Gäste, meine sehr verehrten Herren, darf ich in erster Linie unsere beiden Schweizer Kollegen, Herrn Huber aus Bern und Herm Kaegi aus Zürich, nennen, die auch hier in Bonn Gäste unserer wissenschaftlichen Erörterungen sind, und die ich sehr herzlich willkommen heiße. Wir begrüßen diesen ertragreichen Kontakt mit unseren Schweizer Kollegen auf das wärmste, und wir dürfen wiederholen, was schriftlich ihnen gegenuiber schon wiederholt geäußert wurde. Die Vereinigung selbst hält ihre Tore auch weiterhin für sie geöffnet, und ich bin überzeugt davon, daB ein EntschluB von Schweizer Kollegen, dieses Tor zu durchschreiten, aufrichtige Zustimmung finden würde, ohne daB, solange das nicht geschieht, diese Gefühle auch unsererseits sich anders als darin äußern könnten, dieses Tor eben weiterhin offenzuhalten.

Unter unsern Gästen begrüße ich mit besonderer Freude Kollegen der Bonner und Kölner Fakultäten, ferner die Kollegen der Zivilrechtswissenschaft, die, wie in Marburg, so auch hier, soweit sie an der Tagungsthematik Interesse nehmen, erschienen sind, und mein Dank gilt umgekehrt den Herren der Zivilrechtswissenschaft, insbesondere Herrn Kollegen Dietz, den ich hier auch begrüßen darf, für die Globaleinladung, die sie uns übermittelten zur Teilnahme an der Zivilistentagung, die morgen in Schlangenbad beginnt und die uns am Sonnabendnachmittag, wenn wir dann noch aufnahmefähig sind, nach entsprechendem Ortswechsel 
einen sicherlich aufschluBreichen Vortrag des Kollegen Siebert aus Göttingen bieten würde über Grenzfragen zwischen dem offentlichen und dem privaten Recht.

Ich begrüße ferner herzlich die Herren Schriftleiter der Fachzeitschriften, die wiederum wie im vorigen Jahr in so dankenswerter Weise über den Verlauf der Tagung der Fachwelt berichten werden, und schließlich gilt mein Grußwort Ihnen, den Mitgliedern unserer Vereinigung, unter Ihnen besonders herzlich auch den österreichischen Kollegen, während ich in diesem Jahre leider wiederum unseren Kollegen Jacobi aus Leipzig nicht unter uns begrüßen kann.

Mein Grußwort muß an dieser Stelle von einer schmerzlichen Uberlegung unterbrochen werden. Wir vermissen in unserer Reihe unseren Kollegen von Mangoldt. Ihnen ist bekannt, meine Herren Kollegen, wie kürzlich unser Kollege von Mangoldt von uns gegangen und wie dieses Ende einen tragischen Abbruch einer segensreichen, wissenschaftlichen Arbeit und einer Arbeit für die Allgemeinheit gewesen ist. Seiner und seiner Verdienste ist gedacht worden in Nachrufen, die unsere Kollegen Schönborn und Leibholz ihm gewidmet haben, unser Kollege Leibholz in dem Vorwort zu dem Kommentar, den der Tote noch hat abschlieBen können. Ich glaube, diesen Gedächtnisworten nichts hinzufügen zu sollen, und danke Ihnen, daB Sie sich durch $\mathrm{Ihr}$ Erheben seiner erinnert haben. Wir werden das Gedenken an den Kollegen von Mangoldt gerade hier an diesem Orte in Bonn in bleibender Erinnerung bewahren. Ich danke Ihnen!

Herzlich begrüßen und gedenken darf ich unserer Lebensjubilare des letzten Jahres seit der Marburger Tagung, darunter Herrn Kollegen Apelt, der in diesem Zeitraum das 75. Lebensjahr vollendete, den wir hier unter uns begrüßen dürfen, ferner Herrn Kollegen Laforet, von dem ich mit Bedauern feststellen muB, daß er nicht unter uns weilt, der ebenfalls das 75 . Lebensjahr vollendete, und dann die Herren Kollegen Wenzel und Schönborn, die beide $z u$ meiner Freude hier weilen, die das 70 . Lebensjahr vollendet haben. Mein Gruß gilt auch dem Kollegen Erich Kaufmann, der zu unserem Bedauern trotz der Gunst des Ortes nicht unter uns weilt. Er hat leider einen kleinen Unfall erlitten und schreibt ein paar Zeilen, die ich hier verlesen darf: ,Zu meinem lebhaftesten Bedauern ist es mir nicht möglich, an der diesjährigen Tagung teilzunehmen. Ich hatte am 4. Oktober einen kleinen Unfall durch Uberfahren von einem Zweirad und soll mich durch Bettruhe bis zum nächsten Samstag unbedingt schonen. Ich bitte Sie, die Kollegen bestens von mir zu grüßen, und wünsche der Bonner Tagung einen guten Verlauf. Erich Kaufmann". Ich glaube, wir erwidern diese Grüße mit herzlichen Genesungswilnschen. 
Meine sehr verehrten Herren! Mir wird gesagt, daß ich diesen Grüßen hinzuzufügen habe einen GruB an den Kollegen Giese. Er hat das 70. Lebensjahr in diesem Zeitraum erreicht. Ich bitte um Nachsicht, wenn ich erst durch Zuruf darauf aufmerksam werde, aber wer Herrn Giese vor sich sieht, wird meinen Lapsus entschuldigen.

Die Besonderheit unserer Tagung liegt darin, meine Herren Kollegen, da $\mathrm{B}$ wir, von der Berliner Grlindungstagung abgesehen, zu einer Wissenschaftstagung zum ersten $\mathrm{Mal}$ in einer Hauptstadt unseres Staates versammelt sind.

Die Bedeutung Bonns als Tagungsort brauche ich schwerlich zu unterstreichen, abgesehen davon, $\mathrm{da} B$ der Ort der Tagung selbstverständlich auch Fragen aufgeworfen hat, die in vielerlei Beziehung Erörterungen zu diesem Thema auslösten. Als Geburtsstätte des Grundgesetzes, als Stadt der heutigen Neugestaltung des Bundes und als Universitätsstadt ganz besonderer Tradition, auf die Herr Friesenhahn hingewiesen hat, vermittelt sich unserer Arbeit hier ein Fluidum und eine Atmosphäre, die, wie ich hoffe, unseren Erörterungen von Nutzen und von Segen sein wird. Es würde reizvoll sein können, ohne daß hier diesem Thema nachgegangen wird, einmal zu überdenken, welche besondere Rolle Bonn gespielt hat als Universitätsstadt seit 1818 für die Entfaltung des Preußischen Staates hier am Rhein, und welche besondere Rolle Bonn heute und wohl noch in weiterer Zukunft spielen wird für die Wiederentfaltung einer deutschen Staatlichkeit nach dem Zusammenbruch im Jahre I945. Wenn unsere Themata, wie Herr Kollege Thoma betont hat, zu diesen Fragen in einer besonderen Beziehung stehen, kann, so glaube ich, sich hier eine positive Synthese von Ort und Gegenstand der Handlung ergeben. Und so darf ich zum SchluB nur wïnschen, daß der genius loci unsere Erörterungen beflügeln möge, zu deren Beginn ich nunmehr den fachlichen Teil als eröffnet erklären darf. 